\title{
(Ré)concilier éclairage urbain et environnement nocturne : les enjeux d'une controverse sociotechnique
}

\author{
Samuel Challéat ${ }^{1}$, Dany Lapostolle ${ }^{2}$ \\ 1 Géographie et aménagement, Université de Toulouse 2 -Jean-Jaurès, UMR MA 104, Dynamiques Rurales (DR), 31000 \\ Toulouse, France \\ 2 Géographie et aménagement, Université de Bourgogne, UMR CNRS 6049, Théoriser et Modéliser pour Aménager (ThéMA), \\ 21000 Dijon, France
}

La question de l'éclairage urbain nocturne est posée publiquement de manière de plus en plus significative, d'abord aux États-Unis puis en Europe. Cantonnée à l'origine au domaine de l'astronomie, cette question pose problème dans différents secteurs : l'environnement, la santé, l'urbanisme, mais aussi et surtout l'énergie... En croisant une approche sociologique avec une approche géographique, les auteurs font le récit d'une controverse environnementale aboutissant, en France, à l'inscription de la notion de pollution lumineuse dans la loi Grenelle et questionnent sa dimension spatiale. Ils montrent les différentes logiques et interprétations, à l'œuvre autour de la distinction entre "pollution » et «nuisance » lumineuses, qui traversent les scènes de négociation sur les processus de normalisation et la mobilisation d'outils de zonage.

Mots-clés :

environnement ; éclairage urbain ; controverse ; espaces ; pollution lumineuse

Résumé - Cet article explique la naissance d'un problème environnemental, la pollution lumineuse, appréhendé comme une controverse sociotechnique. Sur une quarantaine d'années, en appui sur la démarche de l'acteur réseau, il retrace les conditions de son émergence, de sa transformation et de sa diffusion aux échelles locales, nationales et transnationales, traversant différents champs professionnels et disciplinaires. Sa mise à l'agenda politique débouche sur des décisions institutionnelles qui peinent à le saisir dans toutes ses dimensions sociale, scientifique et spatiale. La dissémination spatiale de la controverse dans les zonages et le processus de normalisation apparaît alors comme une réponse régulatrice partielle et segmentée à ce problème.

Keywords:

environment; urban lighting; controversies; spaces; light pollution

\begin{abstract}
Reconciling urban lighting and the nocturnal environment: the issues of a sociotechnical controversy. Our paper explains the birth of an environmental problem, i.e. light pollution, viewed as a socio-technical controversy. Supported by the actor-network approach, it traces over forty years the conditions of its emergence, transformation and dissemination to local, national and transnational levels and through various professional disciplines. Schematically, "environmentalists" uphold a holistic approach of "nocturnality" and define artificial light as a pollutant. Facing them, the "technicist" defends a segmented approach and defines artificial light as a nuisance. The introduction of this controversy into the political agenda leads to institutional decisions that grasp with difficulty all its social, scientific and spatial dimensions. The spatial spread of the controversy in the zoning and standardization process appears as a partial and segmented regulatory response to this problem.
\end{abstract}

\section{Introduction}

Kitt Peak, Arizona, États-Unis, 1972. Les astronomes professionnels de l'observatoire national américain de Kitt Peak, cherchant à protéger l'objet de leur activité scientifique, le ciel noir étoilé, contribuent à la réglementation de l'éclairage public de la ville voisine de Tucson.
Comme de nombreuses villes nord-américaines, Tucson connaît depuis le milieu du $X{ }^{\mathrm{e}}$ siècle des mutations morphologiques rapides. Le développement massif de l'automobilité, l'étalement urbain et les faibles coûts de production de l'électricité engendrent l'installation d'un éclairage public toujours plus puissant, augmentant la taille et l'intensité du halo lumineux. D'une gêne perçue

Auteur correspondant : S. Challéat, samuel.challeat@univ-tlse2.fr 
par les astronomes depuis l'observatoire de Kitt Peak, l'idée d'une "pollution lumineuse » émerge et commence à diffuser dans différents réseaux et instances de l'astronomie et de l'éclairagisme. En 1976, l'Union astronomique internationale, réunie en congrès à Grenoble, adopte une résolution pour la protection des sites astronomiques. À la suite, une collaboration avec la Commission internationale de l'éclairage débouche en 1980 sur des recommandations pratiques visant à diminuer le halo lumineux à proximité des observatoires. Les astronomes amateurs entrent dans la danse et relaient localement ces préoccupations professionnelles. En 1993, ils se fédèrent autour de la rédaction d'une charte pour la préservation de l'environnement nocturne. Cette mobilisation se traduit, notamment en France, par la création du Centre pour la protection du ciel nocturne qui deviendra, en 1998, 1'Association nationale pour la protection du ciel nocturne. Ce problème va rapidement intéresser une pluralité d'acteurs aux intérêts et légitimités diverses. Écologues et médecins montrent les effets perturbateurs de la lumière artificielle sur les comportements et les rythmes biologiques. Certaines collectivités locales soucieuses d'économie d'énergie et de leur image environnementale «bricolent » des plans lumière. Les fabricants de luminaires, intégrant ces enjeux, proposent des éclairages publics plus efficients. Enfin, quelques concepteurs lumière invitent à la redécouverte d'une part d'ombre dans l'éclairage urbain.

Pourtant, derrière ces convergences demeurent des conflits d'interprétation sur les impacts réels de la lumière artificielle : pollution ou nuisance lumineuse ? Le choix des termes détermine l'étendue de la controverse (Alkrich et al., 2006). D'un côté, «les environnementalistes», tenants d'une approche globale de la " nocturnité ", définissent la lumière artificielle comme altéragène d'un actif naturel - le noir - et comme polluant à part entière. De l'autre côté, les «technicistes», partisans d'une approche segmentée, qualifient de nuisances les problèmes soulevés par la lumière artificielle. Ce face à face schématiquement présenté permet, à partir d'une approche fondée sur la théorie de l'acteur réseau, d'appréhender les logiques $\mathrm{d}$ 'action qui structurent les controverses sociotechniques liées aux «pollutions/nuisances » lumineuses. Celles-ci « vont bien au-delà des seules questions techniques. Un de leurs enjeux est [...] d'établir une frontière nette et largement acceptée entre ce qui est considéré comme indiscutablement technique et ce qui est reconnu comme indiscutablement social. [...] Reconnaître sa dimension sociale, c'est redonner une chance [à un dossier] d'être discuté dans des arènes politiques » (Callon et al., 2001).

\section{Problématique}

Depuis son entrée dans les arènes politiques dans les années 1970, nous voudrions montrer comment la portée de ce problème de protection du ciel nocturne ${ }^{1}$ se transforme dans la durée, à différentes échelles territoriales, en élargissant les coalitions à de nouveaux acteurs et objets (Gerston, 2004). La conjonction de ces éléments constitue une "fenêtre d'opportunité politique » (Kingdon, 1984) qui débouche sur des décisions institutionnelles. Aussi, la mise à l'agenda politique de cette controverse sociotechnique suppose-t-elle que l'on questionne les logiques de médiation à l'œuvre, les logiques de territorialisation des problèmes et la nature des ressources mobilisées par les acteurs. En d'autres termes, il s'agit d'explorer dans ces controverses les rapports sociaux qui sous-tendent la construction de l'espace, dans le sens où l'espace est porteur et producteur de normes qui en réglementent l'usage et induisent des comportements conformes aux valeurs dominantes dont il est l'expression.

\section{Méthode et matériau empirique}

Pour faire apparaître les dimensions spatiales des controverses, l'analyse s'appuie sur le croisement de sources variées. Entretiens, observations participantes constituent les sources de première main, complétées par le recours à des archives privées, à la littérature grise, à la comparaison d'argumentaires autour de questions saillantes qui déterminent les enjeux des controverses. L'étude de la création et de la circulation d'objets tels que la cartographie des émissions lumineuses, des normes nationales et internationales renseigne sur les jeux d'alliance, le filtrage des acteurs et la qualification des problèmes qui en découlent. Avec l'approche descriptive et interactionniste de la méthode de l'acteur réseau ${ }^{2}$ nous définissons les mécanismes par lesquels se fixent l'identité des acteurs, les rôles qu'ils jouent et les sujets qu'ils abordent. L'élargissement des argumentaires, la circulation d'idées, de cartes, d'études scientifiques, via des portails Internet interactifs, créateurs d'espaces et réseaux transnationaux enrichissent la notion même de nuisance pour la dépasser, la transformant ainsi en un contenant de controverses environnementales dont les contenus se renouvellent avec l'élargissement des coalitions d'acteurs et les asymétries de pouvoir en leur sein. C'est donc par l'étude des connexions d'espaces et

\footnotetext{
1 Cet article prolonge et actualise les travaux de doctorat de S. Challéat (2010) en les augmentant d'une approche des controverses sociotechniques, par le recours à la démarche de l'acteur réseau et la prise en compte du processus de normalisation en cours.

2 Nous considérons l'acteur réseau davantage comme un ensemble d'approches méthodologiques, utiles à la lecture et mise au jour des différents liens entre actants pris dans des complexes organisés, que comme une théorie construite sur des concepts articulés. En conséquence, c'est l'esprit de la démarche plutôt que la lettre que nous retenons.
} 
d'acteurs, des logiques de médiations que la qualification tantôt de nuisance, tantôt de pollution lumineuse sera abordée.

Aussi, dans un premier temps, s'agit-il de définir dans le temps long les conditions d'émergence de la controverse pour ensuite montrer comment celle-ci se projette dans la normalisation de l'espace par l'éclairage public.

\section{Émergence et définition de la controverse}

\section{L'échec d'une innovation politique...}

En 1982, Michel Crépeau, ministre de 1'Environnement, lance les États régionaux de l'environnement. Dans le cadre de la collectivité territoriale nouvellement instituée, les militants associatifs sont invités à définir une charte de l'environnement. L'objectif politique affiché est ambitieux. Il constitue un tournant dans l'élaboration des politiques publiques. La région, par ses missions de planification, d'orientation et de conseil, assoit sa légitimé en s'ouvrant aux associations. La démarche revendiquée est décentralisée et participative ${ }^{3}$. Elle donne la possibilité aux « associations de développer des actions contractuelles de gestion des espaces et ressources naturels ${ }^{4}$. Saisissant cette opportunité politique, les associations astronomiques amateurs entrent en scène, investissent les secrétariats des États régionaux de l'environnement chargés de la rédaction d'un Livre blanc. Elles nomment des référents qui revendiquent la protection du ciel étoilé et parviennent parfois à occuper une position stratégique. C'est par exemple le cas en Bourgogne, où Jean-Claude Merlin, président fondateur de la Société astronomique de Bourgogne, lauréat en 1982 de la Fondation Bleustein Blanchet pour la Vocation, créateur de l'observatoire astronomique au Creusot, découvreur de comètes, devient correspondant des États régionaux de l'environnement de ce territoire.

\section{Poursuivie par les acteurs sociaux}

Las, la charte de l'environnement présentée sur la base des livres blancs régionaux ne verra jamais le jour faute de véritable soutien politique au ministre de l'Environnement. Cependant, l'absence de décision politique ne convainc pas de l'inanité de cette forme d'action

\footnotetext{
3 « On ne forme pas l'opinion à Paris, ni par la télévision seulement, mais à partir de chaque commune, de chaque école, de chaque association ». Consultation nationale des associations de l'environnement, le 23 octobre 1981 à Paris. Déclaration de Michel Crépeau, ministre de l'Environnement, devant les associations, sur la politique de l'environnement : http:/ / discours. vie-publique.fr/notices/813152600.html.

4 Actualité et Environnement, n 8 du 21 janvier 1982.
}

publique (Hirschman, 1991). Cette ouverture de l'action publique est l'occasion pour les associations d'astronomes amateurs de publiciser leurs revendications, de poser les jalons d'une expertise et de constituer un réseau national dont l'action se cristallise en 1993 dans la rédaction d'une «Charte pour la préservation de l'environnement nocturne ». Les principales associations d'astronomie amateur françaises soutenues par des «figures médiaticoscientifiques »-Jacques-Yves Cousteau, Albert Jacquard et Hubert Reeves - y formulent des recommandations pratiques recouvrant les champs de l'urbanisme, de la sécurité des biens et des personnes, de l'économie d'énergie et de leur gouvernance.

Le congrès de Rodez des 7 et 8 octobre 1995 renforce cette entreprise et débouche sur la création d'un comité associatif ouvert aux institutions telles que l'Ademe, EDF et l'Association française de l'éclairage (AFE). L'élargissement de cette coalition va de pair avec la diversité des objets artisanalement bricolés visant à faire connaître la problématique au plus grand nombre : tracts manuscrits, photographies amateurs, embryons de cartographie de ces nouvelles formes de nuisances non répertoriées par les pouvoirs publics, argumentaires et schémas techniques à destination des élus. Le schéma dressé en bas à droite de la figure 1 se rapproche ainsi des normes graphiques employées par les techniciens et les industriels de l'éclairage (représentation en coupe des luminaires et des flux de lumière). On peut y voir une forme de revendication d'un droit à l'expertise de la part des défenseurs du nocturne, qui recourent au même « langage » technique que les professionnels.

En 1998 se tient un second congrès à Rodez à la suite duquel est créée l'Association nationale pour la protection du ciel nocturne (ANPCN), dont les buts statutaires sont « de restaurer et de défendre la qualité du ciel nocturne et de sensibiliser les acteurs concernés pour que soient adoptées les mesures appropriées, au plan national et international ». La stratégie d'internationalisation de la controverse se traduit d'abord par l'adhésion de l'ANPCN à l'International Dark-Sky Association (IDA). Cette dernière, créée en $1988^{5}$ à l'initiative de l'astronome de Kitt Peak, David L. Crawford ${ }^{6}$, acteur-clé de l'accord sur la réglementation de l'éclairage de Tucson, a construit un savoir-faire politique reconnu lui permettant aujourd'hui de disposer d'un " office of Public Policy and Government Affairs " 7 à Washington D.C. L'antériorité de l'association nord-américaine est un atout. À l'origine du "Dark-Sky movement», en pointe dans la protection du ciel nocturne, elle a constitué un corpus documentaire de travaux amateurs et scientifiques ainsi

\footnotetext{
5 En 2011, l'IDA compte environ 11000 membres dans 70 pays.

6 Sa biographie est en ligne sur: http://www.starlight 2007.net/pdf/CV/DavidLCrawford.pdf.
} 


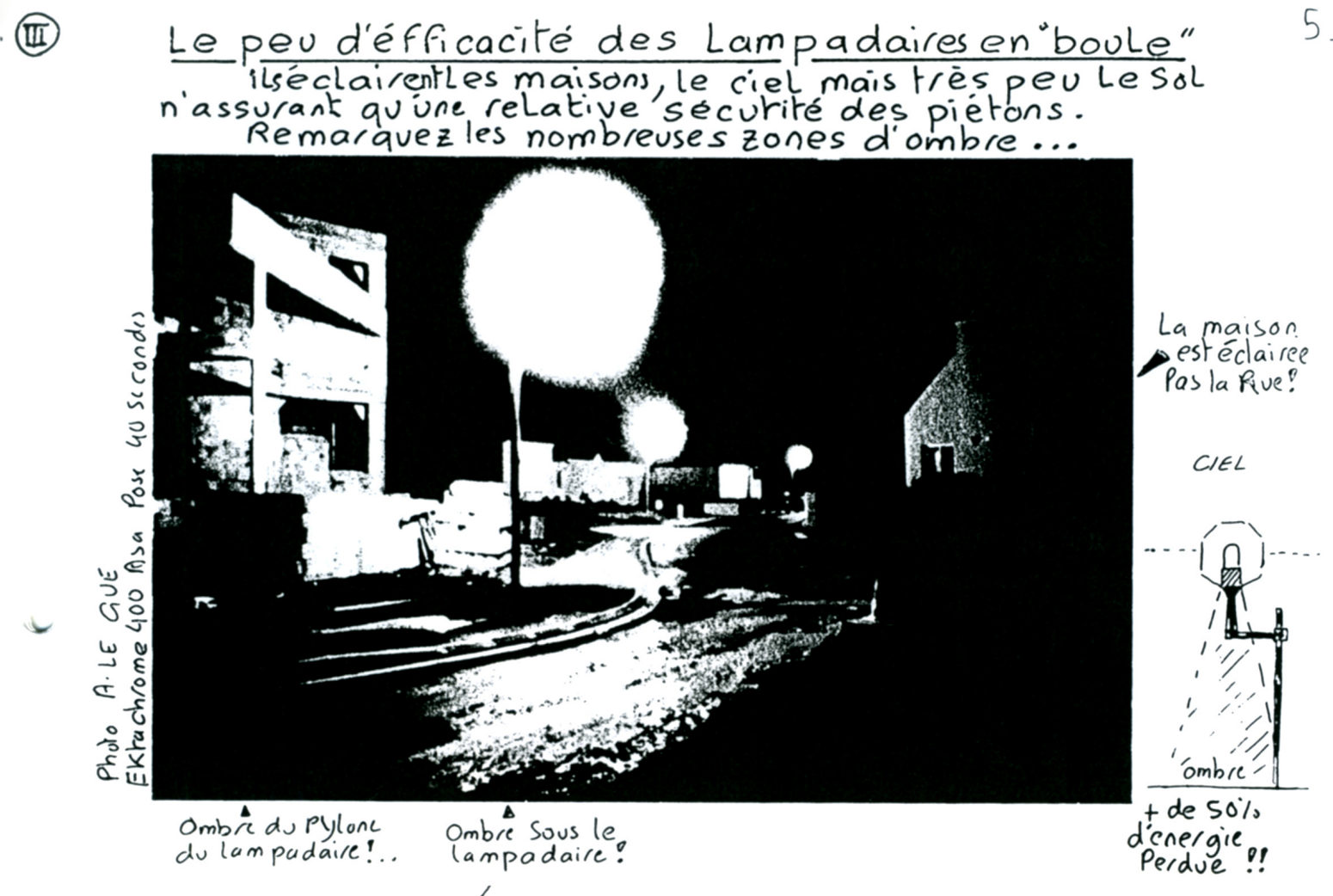

Fig. 1. Extrait d'une documentation de sensibilisation produite par les défenseurs du nocturne (source : archives de la Société astronomique de Bourgogne, Dijon, 1994).

que de préconisations techniques qui sont autant de ressources pour les autres associations nationales moins structurées. Elle a notamment essaimé sa méthode de production participative d'une expertise sur la nocturnité, entendue comme une ressource cognitive mobilisée par différents acteurs, poussés par des motifs pluriels (Dumoulin et al., 2005) «en réponse à des situations confuses appelant une décision d'attribution ou de validation " (Trépos, 1996). Accueillant et formalisant les connaissances vernaculaires et amateurs, cette expertise infuse la production de savoirs scientifiques et institutionnels qui entretiennent et publicisent la controverse. Cette production de nouveaux savoirs scientifiques est validée suivant les canaux habituels de publication et de confrontation aux pairs. Elle légitime en retour cette forme de production participative de l'expertise, les questions de l'indépendance de l'expertise, de son

\footnotetext{
7 Le 2 mars 2009, l'International Dark-sky Association ouvre un bureau à Washington D.C. Son objectif (traduit par nos soins) est de « crée[r] une présence immédiate auprès des organisations et du Congrès dans l'élaboration des normes, tout en générant des possibilités accrues pour sensibiliser à la pollution lumineuse et promouvoir la protection du ciel nocturne » (source : http:// darksky.org/assets/documents/PR/PR_WashingtonDC_final $\% 20(2)$.pdf).
}

impureté scientifique (Memmi, 1989) ou de la politisation des sciences (Saint Martin, 2006) perdant ici de leur acuité.

En 2001, Cinzano, Falchi et Elvidge publient dans un numéro de Monthly Notices of the Royal Astronomical Society un article qui fera date aux niveaux scientifique et militant. Dans «The first world atlas of the artificial night sky brightness », Cinzano et ses collègues (Cinzano et al., 2001) montrent que «sur la base d'une vision moyenne, environ un cinquième de la population mondiale, plus des deux tiers de la population des États-Unis et plus de la moitié de la population de l'Union européenne ont déjà perdu la possibilité de voir la Voie lactée à l'œil nu ${ }^{8}$. Ils produisent un article scientifique dont les associations de défense de la nuit reprennent les illustrations (Fig. 2), participant ainsi à la construction d'une iconographie de la pollution lumineuse, laquelle sera complétée par les images satellitaires de la Terre vue de nuit de la NASA (National Aeronautics and Space Administration), largement diffusées dans le grand public.

\footnotetext{
8 C'est nous qui traduisons : «Assuming average eye functionality, about one-fifth of the World population, more than two-thirds of the United States population and more than one half of the European Union population have already lost naked eye visibility of the Milky Way. "
} 


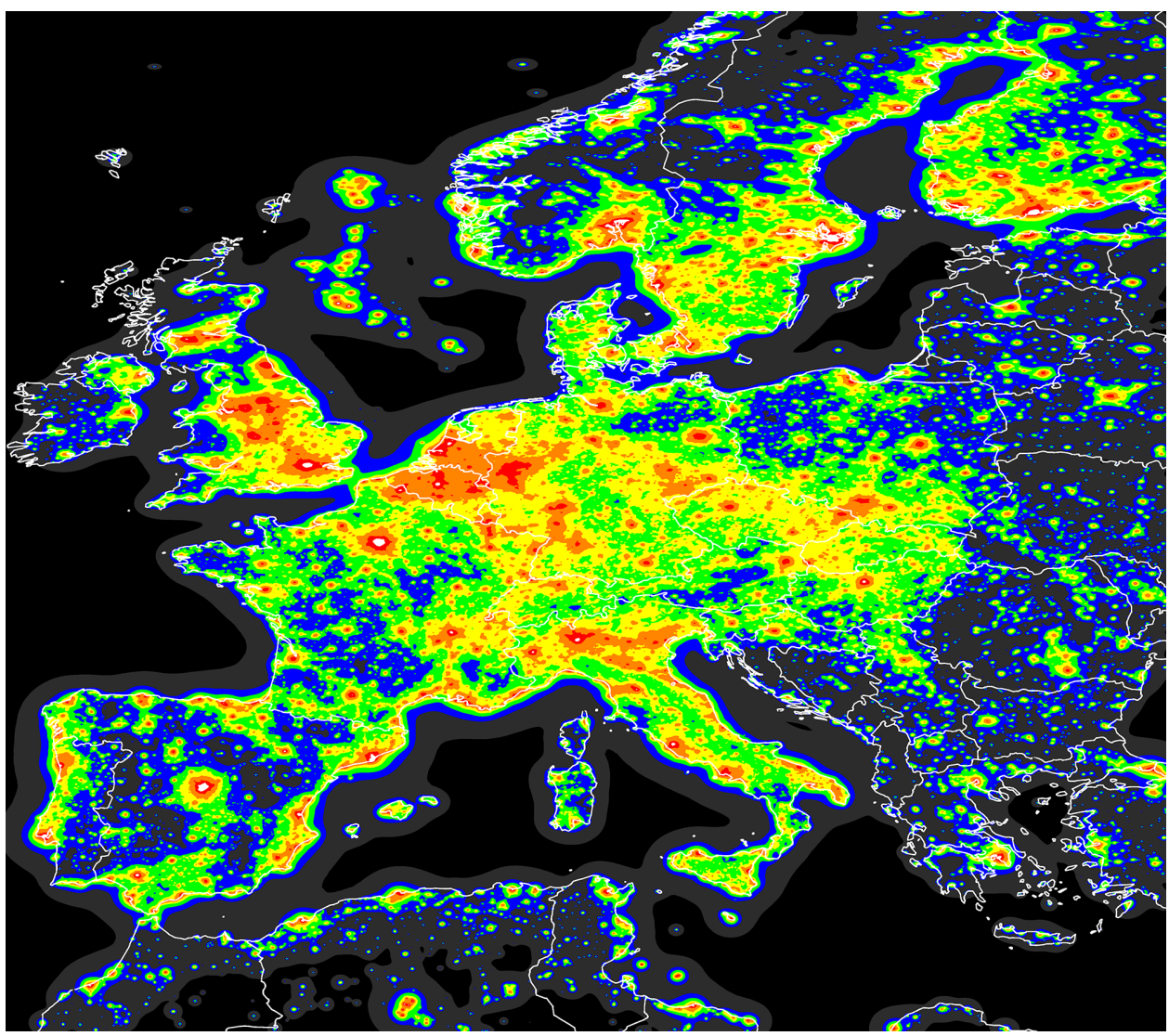

Fig. 2. Cartographie extraite de «The first world atlas of the artificial night sky brightness » (Cinzano et al., 2001) montrant la luminosité artificielle du ciel européen (source: P. Cinzano, F. Falchi [University of Padova], C.D. Elvidge [NOAA National Geophysical Data Center, Boulder]. Copyright Royal Astronomical Society. Reproduced from the Monthly Notices of the RAS by permission of Blackwell Science).

Alertés par ces impacts sur le ciel nocturne, les écologues, quant à eux, mettent en avant les coûts environnementaux de l'éclairage, montrant des écosystèmes fortement perturbés par la lumière artificielle. De nombreux effets sont observés sur la faune par les biologistes à plusieurs échelles. Ces effets relèvent essentiellement, à échelle fine, de mécanismes d'attraction et de répulsion par les sources lumineuses, ainsi que de perturbations, à échelle plus large, d'espèces désorientées par les halos lumineux. De nombreux troubles comportementaux sont également relevés sur les communautés et les écosystèmes à des échelles de temps variables. Du point de vue de la santé humaine, la recherche médicale montre que l'alternance naturelle du jour et de la nuit est le premier donneur de temps pour l'horloge interne de l'Homme. Ce synchronisateur exogène régule la rythmicité circadienne de sécrétion de plusieurs hormones, notamment de la mélatonine. Une désynchronisation de sa sécrétion peut générer stress, fatigue, dégradation de la qualité du sommeil, irritabilité ou troubles de l'appétit. Enfin, la bonne qualité de la rythmicité circadienne de sécrétion de la mélatonine pourrait freiner l'apparition de certains cancers.
La convergence de différentes disciplines scientifiques débouche sur la scotobiologie ${ }^{9}$ définie comme l'étude des réactions biologiques et des comportements nécessitant l'obscurité pendant leur fonctionnement normal (Bidwell, 2010). Au-delà de l'intérêt scientifique de ces travaux, la scotobiologie peut être vue dans sa dimension stratégique et de politisation du public. Selon Bidwell (traduit par nos soins) :

«Il est toujours difficile de recueillir des fonds pour les programmes de protection des écosystèmes. Il est nécessaire de viser tout à la fois l'intérêt public et scientifique des problèmes pour qu'ils soient traités efficacement. Ce sont des problèmes tels que la mort d'oiseaux et les floraisons précoces qui ont suscité l'intérêt du public pour la pollution lumineuse et les réserves de ciel noir. [...] Tout doit être fait de manière à attirer l'intérêt du public. Si cela est fait correctement, la scotobiologie se concentrera de plus en plus

\footnotetext{
9 Le terme scotobiologie est utilisé pour la première fois lors $\mathrm{du}$ symposium international The Ecology of the Night: Darkness as a Biological Imperative, Muskoka, Canada, 22-24 September 2003. Les actes sont en ligne sur le site: www.muskokaheritage.org/ecology-night/.
} 
efficacement sur les véritables conséquences de la pollution lumineuse, et apportera peut-être une révolution dans la façon dont notre société se saisit de cette préoccupation écologique globale. De toute évidence, sans éducation du public, aucune action politique ne sera engagée. Nos parcs peuvent et doivent être conçus comme une opportunité pour éduquer le public » (Bidwell, 2010).

L'éducation politique du public est aussi revendiquée par Globe at Night. La NASA est à l'origine de ce programme éducatif américain institué en 2005 qui met l'accent sur l'enseignement scolaire des sciences. Piloté par le NOAO (National Optical Astronomy Observatory), il s'internationalise en 2009 à l'occasion de l'année mondiale de l'astronomie. Il mise sur une pratique ancienne de la science citoyenne ou science participative qui retrouve sa pertinence dans les controverses environnementales. On peut la considérer comme une mobilisation d'amateurs éclairés au profit de la production de connaissances scientifiques, conçue comme une fin en soi. Mais elle peut aussi être abordée comme l'engagement d'amateurs éclairés dans la production de politiques publiques qui abordent des questions d'ordre scientifique et technique, et l'engagement des chercheurs dans le processus démocratique (Lewenstein, 2004). On se rapproche d'une régulation pragmatique de l'action publique (Habermas, 1973) qui questionne les relations entre décision politique, savoirs techniques et scientifiques. Ainsi la scientifisation du politique repose sur une interrelation critique entre scientifiques et politiques qui conduit non seulement à traduire le savoir scientifique en savoir pratique, mais aussi à rationaliser scientifiquement la domination politique en l'ouvrant via des procédures ad hoc à l'opinion publique. L'arsenal collaboratif, ce travail hors laboratoire en opposition à la science confinée (Callon et al., 2001) mis en place par le programme Globe at Night, l'International Dark-Sky Association ou encore l'Association nationale pour la protection du ciel et de l'environnement nocturnes (ANPCEN), contribue peut-être à l'avènement d'une régulation pragmatique. Mais celle-ci doit s'accommoder des rapports de forces institués.

\section{Les intérêts institutionnalisés des professionnels de l'éclairage}

Historiquement, l'éclairage public est un objet d'aménagement urbain. Cet extrait du code de bonne pratique d'éclairage public et de signalisation lumineuse édité par EDF en 1958 en témoigne :

« Pour les voies urbaines, à l'exigence d'un éclairage efficace s'ajoute désormais celle d'une ambiance lumineuse agréable [...]. Le niveau d'éclairage réalisé et le caractère de l'installation doivent évidemment s'inspirer de la classe de voie et du trafic de voitures et de piétons qui la parcourt. Dès lors, l'éclairage public d'une agglomération doit faire l'objet d'un plan d'ensemble qui s'intègre au plan d'urbanisme. Les voies majeures à grande circulation doivent, par un éclairage magnifique, dessiner l'ossature de la ville [...]. Quant aux monuments historiques, aux parcs publics, aux beaux sites, dont la ville est justement fière, leur mise en valeur devra être particulièrement soignée, et réalisée de manière à constituer aux yeux des promeneurs, de véritables tableaux où les jeux d'ombres, de lumières, de couleurs, et les rapports de luminance (inconscients pour le spectateur, mais scrupuleusement étudiés) concourront à révéler l'âme des choses, et à créer l'émotion recherchée. »

Derrière ce souci esthétique et sensoriel du rapport à la nuit urbaine se cache une approche plus fonctionnaliste de l'éclairage public. Dès 1930, il est rationalisé pour être appliqué de façon uniforme dans un espace urbain réduit à un système de circulations et stationnements. À l'augmentation du trafic nocturne des années 1960, répond celle de la quantité de lumière artificielle le long des voies. Cette approche fonctionnaliste s'accompagne de prescriptions. En 1961, l'AFE fait paraître les premières Recommandations relatives à l'éclairage des voies publiques ${ }^{10}$, suivies des premières Recommandations internationales de la Commission internationale de l'éclairage (CIE) en $1965^{11}$.

\section{Du monopole à la pluralisation de l'expertise : les technicistes s'opposent aux environnementalistes}

L'AFE, fondée en 1930, regroupe aujourd'hui «plus de 1000 architectes, urbanistes, concepteurs, décorateurs, médecins, chercheurs, ophtalmologistes, ingénieurs des villes, fonctionnaires de l'équipement routier et urbain, installateurs, distributeurs d'énergie électrique, grossistes distributeurs, fabricants de lampes, de luminaires, de systèmes de gestion et de composants ${ }^{12}$. La diversité de ses adhérents lui assure une position incontournable dans l'expertise des usages de l'éclairage et de la normalisation des moyens de production et de contrôle de la lumière.

Pour relayer son action, elle utilise deux leviers: d'une part, les centres régionaux, en direction des services techniques urbains et des syndicats intercommunaux de l'énergie, et d'autre part, la revue $L u x$, créée en 1928, qui demeure une des principales publications de l'éclairagisme. Enfin, l'AFE publie régulièrement des recommandations: Efficience énergétique en éclairage

\footnotetext{
10 AFE, 1961, Recommandations relatives à l'éclairage des voies publiques, Paris, Lux Éditions.

11 CIE, 1965, International recommendations for the lighting of public thoroughfares, Paris, CIE.

12 Site Internet de l'AFE, http:/ / www.afe-eclairage.com.fr/.
} 
public, Guide de l'éclairage des installations sportives, etc. Leur lecture laisse transparaître les dilemmes auxquelles l'association est confrontée en raison de la pluralité des intérêts, des savoirs et des représentations des acteurs qu'elle regroupe. "Éclairer juste», c'est intégrer la contrainte environnementale dans la logique économique de nombreux professionnels de l'éclairage.

«Moije pars du fait quel'AFEc'est une association d'éclairagistes / de gens / de professionnels / des professionnels de l'éclairage donc qui ont/ qui ont euh / entre guillemets une manne financière à se faire sur l'éclairage / donc on peut pas être à mon avis objectif quand il y a del'argent en jeu [...] c'est difficile d'être juge et partie en fin de compte / on peut pas dire euh "on réduit l'éclairage" mais bon si on réduit l'éclairage moi j'en vends plus d'éclairage / euh et je pense qu'ils ne se posent pas aussi les bonnes questions c'est-à-dire qu'on va se dire "ben moi je ne vends plus d'éclairage" au lieu de se dire "ça me permettrait de rénover un parc complet" / / je pense quel'AFE le problème c'est que euh / c'est que c'est/ ils / ils / ils sont / c'est / c'est pas assez euh / entre guillemets pluriactivités il va y avoir des industriels des éclairagistes mais il ne va pas y avoir par exemple des personnes d'une association » (entretien employé syndicat d'énergies, 2009).

« Parce que j'ai reçu le président de l'AFE là / je leur ai dit froidement "dites donc qui fait la norme?" Il me dit "ben c'est moi" / je dis "qui êtes-vous, vous ?" "je suis fabricant" / je dis "effectivement !" / moi j'ai connu ça euh dans l'eau potable euh où c'étaient les fabricants de stations d'eau potable qui faisaient la norme donc une fois que vous aviez trouvé les nitrates c'est très bien ils vous trouvaient autre chose puis autre chose et vous n'aviez jamais fini » (entretien directeur syndicat d'énergies, 2007).

En 2010, 1'AFE édite dans Lux une plaquette intitulée «Éclairage public : Réponses à 40 questions trop souvent dévoyées ». À la première question posée : «Les nuisances dues à la lumière artificielle en éclairage public peuvent-elles être qualifiées de «pollution lumineuse »? », la réponse apportée par 1'AFE marque ses distances avec l'ANPCEN. Sa réponse est :

«NON - Une pollution concerne simultanément les hommes, les animaux, les végétaux, là où elle se produit et son traitement ne peut être que long et unique comme celui de toutes les pollutions telles celles de l'air ou de l'eau, par exemple. La lumière ne pollue pas, la lumière est invisible ; mais elle peut générer des nuisances multiples et variées dont les effets, autant que les remèdes sont spécifiques et différents dans chaque cas. Dès que la lumière cesse, ses nuisances éventuelles disparaissent. »

\section{L'ANPCEN réplique :}

«OUI - Car la lumière en excès envoyée en dehors de la zone utile à éclairer et lorsqu'elle dépasse un certain seuil (en terme de flux et composition spectrale) altère l'environnement nocturne tout d'abord via un effet de répulsion ou $\mathrm{d}$ 'attraction sur la faune nocturne suivant l'espèce considérée et en modifiant les cycles d'alternance jour/nuit avec un effet sur la flore et le sommeil chez l'homme via la lumière intrusive. $» 13$

L'opposition est prégnante entre une approche segmentée, qualifiée de «techniciste » qui aborde les problèmes sous le seul angle des nuisances, et une approche globale, nommée « environnementaliste » qui désigne par « pollution » les impacts de la lumière artificielle. Au-delà des enjeux sémantiques et scientifiques, $c^{\prime}$ est la voix au chapitre de l'expertise qui est en question. En témoigne cet extrait d'une publication de l'AFE ("Polémiques sur la norme expérimentale Afnor "Nuisances lumineuses": quand l'outrance nuit à la vérité », 2011) : "Lorsque l'information ne permet pas de convaincre et d'influencer le citoyen dans le sens désiré, certains groupes de pression n'hésitent pas à promouvoir des campagnes de désinformation accumulant sans scrupules des contre-vérités, des erreurs techniques indétectables par des non-spécialistes, en s'appuyant sur les domaines sensibles du moment (environnement et énergie). »

La négation de la pollution lumineuse est aussi le fait de quelques concepteurs lumière. L'un des plus réputés, Roger Narboni, affirme dans la revue Urbanisme : «On essaye de faire comprendre aux citadins lambda que s'il y a de la pollution lumineuse dans nos villes, c'est d'abord parce qu'il y a de la pollution. Parce que la lumière, c'est immatériel, cela ne se voit que dans la matière ou les éléments en suspension » (Narboni, 2011).

Cette volonté de déplacer la pollution lumineuse vers la pollution atmosphérique est une stratégie d'évitement - voire d'évidement - de la controverse d'expertise. Elle illustre la difficulté de définir termes, unités de mesure, quantifications des effets et impacts nécessaires à l'universalisation du problème et de sa gouvernance.

\section{Participation et universalisation du problème : faire de la protection du ciel nocturne une question environnementale}

Dès sa naissance, il est reproché à l'ANCPN d'être une simple émanation du mouvement associatif astronomique. Elle change de nom, devient l'ANPCEN en 2006 et s'oriente vers une défense globale de l'environnement nocturne, se donnant ainsi un droit d'accès aux négociations du Grenelle de l'environnement de 2007. Paul Blu, président de l'association, précise : «Nous devons cesser de prêter le flanc au rejet de nos arguments provoqué par un nom qui met d'abord en évidence la protection du ciel nocturne. Pour y parvenir, nous devons modifier

\footnotetext{
13 ANPCEN, 2010, « Les réponses de l'ANPCEN aux 40 questions soulevées par l'AFE sur l'éclairage public» (http:// www.parc-vosges-nord.fr/medias/File/_doc_dyn/french/ 4reponses_aux-40question-afe1330097954.pdf).
} 
le nom de l'association et lui donner une connotation environnementale $» 14$.

Poursuivant cette stratégie, elle entreprend une action de « green washing » et adopte un nouveau logotype évoquant « l'engagement, la veille attentive, la protection, le rassemblement dans un cadre évoquant le danger de notre situation (les dangers menaçant notre environnement nocturne) et la capacité à apporter des solutions ${ }^{15}$. À l'automne 2010, Anne-Marie Ducroux remplace Paul Blu à la tête de l'association. Elle n'est pas issue du sérail astronomique. Son parcours professionnel dans le monde de la communication et des médias, son engagement personnel dans la création du Conseil national du développement durable qu'elle préside entre 2005 et 2006 sont des ressources relationnelles pour l'ANCPEN. Cette personne fait le lien avec les ONG généralistes et le monde politique. Son entregent, sa connaissance de la fabrication des lois et du fonctionnement de l'administration font d'elle une médiatrice qui permettra à l'association de rester dans le jeu post-Grenelle. Mais c'est d'abord l'adhésion à France nature environnement (FNE) en 2007 qui force les portes du Grenelle. FNE fédère près de 3000 associations environnementales au niveau national et synthétise l'expertise réalisée par les associations. Elle s'est rendue incontournable dans les principales commissions nationales de concertation environnementale. L'adhésion de l'ANPCEN à cette structure la fait entrer dans un réseau lui donnant une légitimité environnementale plus forte au niveau national. La protection du nocturne trouve ainsi sa place à la table des négociations du Grenelle fin 2007.

Lors de la première phase de négociation, les ONG occupent une place de choix aux côtés de l'État, des collectivités locales, des employeurs et salariés. Elles participent aux six groupes de travail rassemblant chacun quarante membres répartis en cinq collèges. La pollution lumineuse portée par FNE est intégrée au projet de loi Grenelle I de 2008 dont l'article 36 dispose : « Les émissions de lumière artificielle de nature à présenter des dangers ou à causer un trouble excessif aux personnes, à la faune, à la flore ou aux écosystèmes, entraînant un gaspillage énergétique ou empêchant l'observation du ciel nocturne feront l'objet de mesures de prévention, de suppression ou de limitation $» 16$.

Le 23 septembre 2008, Nathalie Kosciusko-Morizet, secrétaire d'État chargée del'Écologie, rassembleà l'observatoire de Paris-Meudon différents acteurs intéressés par

\footnotetext{
14 SOS, bulletin de l'ANPCEN, avril, mai et juin 2006.

15 SOS, bulletin de l'ANPCEN, numéro 37, printemps 2008.

16 L'article 36 du projet de loi Grenelle I est le débouché politique majeur attendu par les associations d'astronomes et de protection del'environnement nocturne. «L'article 36 » devient l'étendard de l'aboutissement institutionnel de leur « lutte».
}

la pollution lumineuse afin de porter « l'article 36 » (qui deviendra l'article 41 dans la loi promulguée le 3 août 2009) à l'attention des médias nationaux :

«Phénomène trop longtemps ignoré, la pollution lumineuse figure désormais dans le projet de loi relatif au Grenelle de l'environnement, examiné au Parlement à partir d'octobre prochain. Cette reconnaissance législative permettra de créer dans le code de l'environnement un nouveau chapitre destiné à réglementer le suréclairage. C'est l'aboutissement d'un combat de longue date pour Nathalie Kosciusko-Morizet, secrétaire d'État chargée de l'Écologie, qui tient à sensibiliser les élus et le grand public à cet enjeu environnemental encore mal connu $» 17$.

Par «combat de longue date », la secrétaire d'État chargée de l'Écologie fait référence à la proposition de loi $\mathrm{n}^{\mathrm{O}} 2275$ relative à la lutte contre les pollutions lumineuses nocturnes, qu'elle avait présentée, alors députée, le 14 avril 2005 à l'Assemblée nationale. Le 29 juin 2010, la Chambre basse adopte le projet de loi portant engagement national pour l'environnement dite « Grenelle II ». L'article 173 inscrit la prévention des nuisances lumineuses dans le Code de l'environnement. Le décret du 12 juillet 2011 y crée dans la partie réglementaire (articles R. 583-1 à R. 583-7) un chapitre spécifique aux nuisances lumineuses. Il définit les installations concernées, le zonage permettant d'adapter les exigences aux enjeux des territoires concernés (agglomération, espaces naturels, sites astronomiques) ainsi que les principales prescriptions techniques qui peuvent être réglementées par arrêté. Le premier texte d'application est signé le 25 janvier 2013. Il concerne à la fois l'éclairage intérieur émis vers l'extérieur des bâtiments non résidentiels (vitrines de commerces, bureaux, etc.) et l'éclairage des façades de ces mêmes bâtiments et encadre les horaires de fonctionnement de ces installations.

Toutefois, la première prise en considération législative et réglementaire de la pollution lumineuse reste liée à la fabrication des normes techniques qui en découlent. La production de seuils d'éclairages révèle la permanence du clivage entre approches techniciste et environnementaliste qui structurent cette controverse.

\section{Les dimensions spatiales de la controverse}

La dimension spatiale de cette controverse apparaît dans les différentes scènes de normalisation entendues comme dispositifs de régulation. Ils contiennent une pluralité de lieux de négociation qu'il faut identifier pour en rendre compte dans leur globalité. Cette

\footnotetext{
17 Dossier de presse sur le déplacement de la secrétaire d'État chargée de l'Écologie à l'observatoire de Paris-Meudon le 23 septembre 2008.
} 
démarche permet d'appréhender les normes législatives, réglementaires et techniques qui, si elles relèvent de scènes, valeurs et légitimités différentes, sont pourtant liées dans leurs effets régulateurs (Thévenot, 1997). De la Commission européenne à la ruelle du village, les scènes de normalisation de l'éclairage public sont traversées d'enjeux divers et pluriels (économiques, techniques, sanitaires, environnementaux) soit autant d'arguments en tension. Par exemple, la normalisation industrielle et commerciale du type Afnor s'accorde difficilement avec les revendications civiques d'ordre environnemental. On retrouve les mêmes questions dans différents problèmes environnementaux comme la production d'indicateurs sur la qualité de l'air ou la pollution des sols. La construction de ces outils de régulation et les débats dont ils sont l'objet indiquent les difficultés à concilier les approches et les méthodes des acteurs qui concourent à la mise en place des outils, mais révèlent aussi les rapports de force dans la définition des normes selon la vision qui prévaut dans un domaine professionnel (Boutaric, 2007). Les interactions qui en découlent nécessitent l'organisation de concertations entre une variété d'échelles institutionnelles, de professions et de milieux intéressés, ainsi que des lieux où puissent se réaliser dialogues, controverses et collaborations. La pluralité des compromis élaborés entre technicistes et environnementalistes débouche sur le zonage qui est une façon de désigner les problèmes et d'énoncer les normes susceptibles de les traiter partiellement, mais surtout de manière segmentée.

Cette dissémination du problème dans l'espace par le zonage participe de la fabrique de la norme. Celle-ci entérine certains espaces et en produit de nouveaux. À l'inverse, l'espace est lui-même producteur de normes.

\section{La controverse autour de la définition de la norme produit de l'espace}

La fabrication de la norme expérimentale Afnor XP X90-013 en application de la loi Grenelle II et de la directive européenne concernant les rayonnements optiques de 2005 est en cours. Son enjeu est de déterminer des seuils et indicateurs de rayonnement lumineux dans l'espace public et privé, pour protéger la biodiversité et la santé des personnes. Mais il est aussi de sécuriser les échanges commerciaux. C'est la raison pour laquelle certains agents économiques intègrent l'activité de normalisation à leur stratégie.

La composition de la commission de normalisation reflète relativement cette pluralité d'intérêts et de valeurs ${ }^{18}$. Si une seule association de protection de

\footnotetext{
${ }^{18}$ La composition de la commission Afnor XP X90-013 est consultable sur :http://www2.afnor.org/espace_normalisation/ structure.aspx?commid $=2404$.
}

l'environnement, l'ANPCEN, est présente, les direction générale du Travail et direction de la Prévention des Pollutions et des Risques pourraient contribuer à dépasser le caractère purement marchand de la normalisation. Sans préjuger des intentions des membres de la commission, sa composition traduit la permanence du clivage entre approche techniciste et environnementaliste. En témoigne cet échange aigre-doux entre l'ANPCEN et l'AFE à propos de la définition des seuils d'éclairage.

La première réclame un seuil maximal à ne pas dépasser. Cette position permettrait à terme l'extinction totale :

«Particulièrement laxiste dans la définition des seuils de puissance lumineuse des installations d'éclairage et réductrice dans les paramètres pris en compte, cette norme expérimentale va à l'encontre des préconisations de scientifiques, et de références internationales pour limiter les nuisances lumineuses atmosphériques utilisées partiellement. Elle pourrait même inciter à l'objectif inverse recherché... Paradoxale, la méthode retenue part du principe d'un éclairage "minimal à maintenir" et non "maximal à ne pas dépasser" pour limiter les nuisances lumineuses. [...] Elliptique, elle élimine de son champ tous les aspects biologiques des conséquences d'un éclairage artificiel excessif ou inadapté ${ }^{19}$.

La seconde préconise un seuil d'éclairage minimal :

«Il faut rappeler qu'il s'agit d'une norme expérimentale et qu'à ce titre, elle ne peut traiter que des sujets "mesurables et vérifiables". C'est pourquoi, écrire, comme le fait l'ANPCEN, que la norme est "inutile" parce qu'elle néglige les conséquences de la lumière sur la biodiversité est inexact car, jusqu'à ce jour, ces effets dus aux éclairages publics ne sont pas mesurables et ne peuvent être intégrés dans une norme expérimentale. [...] L'éclairage public est en pleine mutation technologique. On sait aujourd'hui faire des économies d'énergie dépassant régulièrement $50 \%$ de l'énergie consommée jusqu'alors. On sait moduler l'éclairage à la demande. En France la production de $\mathrm{CO}_{2}$ ne concerne pratiquement pas l'éclairage public alimenté à $85 \%$ par le nucléaire ; l'éclairage public n'est pas non plus gravement concerné par la pointe de consommation, puisqu'il n'en représente que $2 \%$ actuellement, alors que la majorité des économies reste à faire $»^{20}$.

C'est ici le rapport des uns et des autres à la connaissance scientifique - actuellement imparfaite en matière d'effets sanitaires, par exemple - qui est remarquable.

\footnotetext{
${ }^{19}$ Extrait du communiqué de presse de l'ANPCEN du 15 juin 2011. «L'Association nationale pour la protection du ciel et de l'environnement nocturnes (ANPCEN) appelle au retrait de la norme expérimentale censée "minimiser" les "nuisances lumineuses extérieures" ».

20 «Polémiques sur la norme expérimentale Afnor "Nuisances lumineuses" : quand l'outrance nuit à la vérité », Le Point de Vue de l'AFE, numéro 13, $1^{\mathrm{er}}$ juillet 2011.
} 
Pour l'ANPCEN, les preuves scientifiques accumulées par les écologues et les médecins suffisent à la prise de décision (principe de précaution) quand, pour l'AFE, le même savoir scientifique laisse encore une place trop importante à l'incertitude pour qu'il puisse fonder cette même décision. Cet usage stratégique de l'état des savoirs scientifiques doit être replacé dans les épreuves qui ont cours lors des processus de normalisation. Même si elles se déroulent sur des scènes différentes, elles sont structurées par l'antagonisme entre les technicistes et les environnementalistes. En rapprochant ou éloignant les acteurs de la controverse, ces épreuves déterminent ce qui doit être pris en compte ou non par la loi et la norme technique. Pour les technicistes, ce qui est mesurable et vérifiable ; pour les environnementalistes, ce qui pourrait advenir.

Leur rapprochement se fait sur les économies d'énergie dans tous les espaces. Il débouche aussi sur un nouveau zonage, les sites d'observation astronomique, localisés dans des espaces à faible densité de population et d'activité, et la prise en compte des nuisances lumineuses dans les zonages environnementaux existants: réserves et parcs naturels, sites classés, etc. Leur éloignement est sensible sur les espaces à forte densité de population et d'activité dans lesquels l'intrication des enjeux sanitaires, environnementaux et économiques est plus marquée. Soit la dangerosité de l'éclairage pour la santé humaine est reconnue, dans ce cas ce sont les zones à forte densité et leurs habitants qui doivent être protégés contre les nuisances lumineuses. Soit la dangerosité de l'éclairage n'est pas avérée avec certitude, et les intérêts économiques se manifestent plus directement. Cela ne signifie pas pour autant l'absence d'effet de la norme : elle dit l'existence d'un risque pour la santé des personnes et sa prise en compte par un dispositif de régulation rassurant pour le grand public.

\section{L'espace produit la norme}

Les objets spatiaux mentionnés par le décret du 12 juillet 2011 (publié au JO le 13 juillet 2011) sont au cœur de la production de la norme d'éclairage. Leurs caractéristiques sont autant d'arguments pris en compte dans la définition des seuils-controverses, ce qui signifie que l'adaptation locale de la norme d'éclairage érige l'espace en médiateur des rapports sociaux. À titre d'illustration, l'expérience conduite dans le département de la Saône-et-Loire entre 2007 et 2009 est riche d'enseignements. LeSyndicat départemental d'énergie de Saône et Loire (Sydesl) en partenariat avec le correspondant local de l'ANPCEN, la direction interdépartementale des Routes, les maires des communes rurales concernées, les chargés de mission des communautés de communes et le laboratoire Théma (UMR CNRS 6049) mettent au point une méthode expérimentale de définition des normes d'éclairage public au regard de différentes caractéristiques des territoires intéressés. La faible densité de cet espace à dominante rurale, la présence d'une faune et d'une flore remarquables ${ }^{21}$, la qualité des aménités paysagères, la sinuosité des routes départementales et rurales sont portées par les différents participants aux ateliers qui se présentent chacun comme le dépositaire $\mathrm{d}^{\prime}$ une fraction du système spatial qui se mue en système d'adaptation située de la norme européenne EN1320122. Ainsi, pour chaque type de voie, des valeurs d'éclairement sont définies qui se rapprochent de la borne inférieure de la fourchette donnée par la norme EN13201. En complément, le recours aux formes de signalisation passive est encouragé afin qu'en matière de sécurité routière, l'éclairage public ne supplante l'attention et la vigilance du conducteur. Une charte accompagne le dispositif. Elle vise l'uniformisation de l'éclairage public dans les communes rurales à partir des zonages Znief, Zico et zones sensibles autour des sites d'observations astronomiques. Suivant les différents zonages, les caractéristiques des luminaires sont déterminées en fonction de leur type, des horaires de coupure de l'éclairage public sont préconisés, et un système de gestion du réseau d'éclairage public limitant la puissance à certaines heures de la nuit est proposé aux communes ne souhaitant pas couper complètement l'éclairage public en milieu de nuit (Challéat, 2009).

Ce système d'adaptation locale de la norme apporte des réponses pratiques aux questions posées par les élus en matière de sécurité des biens, des personnes et des déplacements tout en intégrant la protection de l'environnement. Ce dispositif, négocié avec les services techniques de l'État, est étendu au département voisin du Jura qui intégrera un panel plus large de participants. Informé de ce projet, le chargé de mission « Maîtrise de la demande d'électricité » à l'Ademe relaie cette initiative au niveau national. Ce travail est présenté lors du piquenique médiatisé de l'observatoire de Paris-Meudon le 23 septembre 2008. Alors qu'il nourrit la controverse lors des négociations publiques du Grenelle II, il reste lettre morte au niveau départemental renforçant paradoxalement le rôle de médiateur social joué par l'espace.

\section{Conclusion}

Cette étude du déroulement d'une controverse sociotechnique qui, sur une quarantaine d'années, traverse des

\footnotetext{
${ }^{21}$ Ce capital écologique spatial est protégé dans des zones naturelles d'intérêt écologique faunistique et floristique et des zones importantes pour la conservation des oiseaux.

22 La norme européenne EN13201, applicable en France depuis 2005, de portée non obligatoire, donne les niveaux d'éclairement minimums à maintenir en fonction du type de voie ou des lieux piétonniers. Elle est en cours de révision depuis 2009.
} 
scènes animées par des réseaux locaux, nationaux et transnationaux, permet de montrer comment les rapports sociaux investissent l'espace et le structurent. La controverse met en scène un grand nombre d'acteurs qui - amenés à créer et acquérir des savoirs - se placent en situation d'expertise pour contribuer au "policy making " (Boutaric, 2007), à la fabrication des normes en particulier. Dans ce jeu, des conflits d'interprétation apparaissent et font évoluer les positions et arguments au fil des changements de qualification du problème abordé.

Certes, on observe que l'État intervient pour institutionnaliser les rapports de force territorialement ancrés, mais cette lecture institutionnaliste ne suffit pas à montrer les mécanismes de prise en considération et de pérennisation d'un problème publique. Pour que la protection du ciel étoilé demeure une préoccupation dans le temps long, de Kitt Peak au Grenelle, les logiques militantes, individuelles et collectives ont élargi et modifié leur répertoire de mobilisation au-delà des frontières (Charlier et Bourgeois, 2013 ; Collectif Renoir, 2014). Le Dark-Sky Movement, l'International Dark-Sky Association, par les usages participatifs et pédagogiques qu'ils font des productions scientifiques comme "The first world atlas of the artificial night sky brightness » ou la campagne Globe at Night, essaiment via les réseaux sociaux des méthodes qui rendent central le travail de visibilité du problème. Celui-ci est alimenté par de nouveaux savoirs scientifiques (scotobiologie, médecine du sommeil, etc.).

Mais si cette condition est nécessaire, elle est insuffisante. Le rôle de certains individus qui font le pont entre différentes échelles et logiques d'action comme Dave Crawford, Nathalie Kosciusko-Morizet, Anne-Marie Ducroux ou encore Jean-Claude Merlin, président fondateur de la Société astronomique de Bourgogne, est essentiel à la visibilité de la controverse. Autrement dit, les relais de mobilisation (Cefaï et Terzi, 2012), qu'ils soient des individus, des groupes, des objets ou des institutions, parce qu'ils traversent les espaces controverses et les structurent doivent être au cœur de l'analyse de ce qui pérennise un problème environnemental et augmente sa considérabilité morale (Goodpaster, 1971). Cependant, la dissémination spatiale de la controverse dans les zonages et le processus de normalisation apparaît comme une réponse régulatrice partielle et segmentée à un problème complexe, de par l'imbrication de ses dimensions sociales, scientifiques et spatiales.

\section{Références}

Alkrich, M., Callon, M., Latour, B, 2006. Sociologie de la traduction : textes fondateurs, Paris, Presse des Mines.

Bidwell, R.G.S., 2010. Scotobiology: The ecology and sociology of light pollution, in Bondrup-Nielsen, S., Beazley, K.,
Bissix, G., Colville, D., Flemming, S., Herman, T., McPherson, M., Mockford, S., O'Grady, S. (Eds), Ecosystem Based Management: Beyond Boundaries. Proceedings of the Sixth International Conference of Science and the Management of Protected Areas, 21-26 May 2007, Acadia University, Wolfville, Canada, 425-428.

Boutaric, F., 2007. Les réseaux de la qualité de l'air : des associations stratèges actrices de l'action publique, Développement Durable et Territoires [En ligne], Varia, mis en ligne le 08 mars 2007 (online: http://developpementdurable. revues.org/2878).

Callon, M., 2006. Pour une sociologie des controverses techniques, in Alkrich, M., Callon, M., Latour, B., Sociologie de la traduction: textes fondateurs, Paris, Presse des Mines, 135-157.

Callon, M., Lascoumes, P., Barthe, Y., 2001. Agir dans un monde incertain: essai sur la démocratie technique, Collection La couleur des idées, Paris, Le Seuil.

Cefaï, D., Terzi, C. (Eds), 2012. L'expérience des problèmes publics: perspectives pragmatistes, Paris, Éditions de l'EHESS.

Challéat, S., 2009. La pollution lumineuse : passer de la définition d'un problème à sa prise en compte technique, in Deleuil J.-M. (Ed.), Éclairer la ville autrement, Lausanne, Presses Polytechniques et Universitaires Romandes, 182187.

Challéat, S., 2010. "Sauver la Nuit»: empreinte lumineuse, urbanisme et gouvernance des territoires. Thèse de doctorat de Géographie, soutenue le 13 octobre 2010, Université de Bourgogne, Dijon.

Charlier, B., Bourgeois, N., 2013. « Half the park is after dark». Les parcs et réserves de ciel étoilé : nouveaux concepts et outils de patrimonialisation de la nature, L'Espace Géographique, 42, 3, 200-212.

Cinzano, P., Falchi, F., Elvidge, C.D., 2001. The first world atlas of the artificial night sky brightness, Monthly Notices of the Royal Astronomical Society, 328, 3, 689-707.

Collectif Renoir (Ressources environnementales nocturnes, tourisme, territoires), Bénos R., Challéat, S. (coord.), Dupuy, P.-O., Girard, F., Lapostolle, D., Michalczak, J., Milian, J., Poméon, T., 2014, travaux en ligne (online: http://renoir.hypotheses.org).

Dumoulin, L., La branche, S., Robert, C., Warin, P. (Eds), 2005, Le Recours aux experts : raisons et usages politiques, Grenoble, Presses universitaires de Grenoble.

Gerston, L.N., 2004. Public Policy Making: Process and Principles, New York, M.E. Sharpe.

Goodpaster, K.E., 1971. On being morally considerable, Journal of Philosophy, 75, 6, 308-325.

Habermas, J., 1973. Scientifisation de la politique et opinion publique, in Habermas, J., La Technique et la science comme idéologie, Paris, Gallimard, 97-132.

Hirschman, A.O., 1991. Deux siècles de rhétorique réactionnaire, trad. de l'anglais par P. Andler, Paris, Fayard, Collection L'espace du politique.

Kingdon, J., 1984. Agendas, Alternatives and Public Policies, Boston, Little Brown and Company.

Lewenstein, B.V., 2004. What does citizen science accomplish?, Prepared for meeting on Citizen Science, CNRS, Paris, 8 juin 2004. 
Memmi, D., 1989. Savants et maîtres à penser, la fabrication d'une morale de la procréation artificielle, Actes de Recherches en Sciences Sociales, 76-77, 98.

Narboni, R. (propos recueillis par T. Paquot), 2011, L'invité Roger Narboni, Urbanisme, 380, 77-84.

Rémy, E., November, V., D'Alessandro-Scarpari, C., 2004. L'«espace» d'une controverse, EspacesTemps.net, Travaux, 18 janvier 2004 (online: http:// www.espacestemps.net).
Saint Martin, D., 2006. Expertise, in Boussaguet, L., Jacquot, S., Ravinet, P. (Eds), Dictionnaire des politiques publiques, Paris, Presses de Sciences Po, 211-220.

Thévenot, L., 1997. Un gouvernement par les normes: pratiques et politiques des formats, in Conein, B., Thévenot, L. (Eds), Cognition et information en société, Éditions de l'EHESS, 205-242.

Trépos, J.-Y., 1996. La Sociologie de l'expertise, Paris, Presses universitaires de France.

Reçu le 12 mai 2013. Accepté le 7 mai 2014. 